\title{
Teaching Anxiety, Stress and Resilience during the COVID-19 pandemic: Evaluating the vulnerability of academic professionals in Mexico through the Adapted COVID-19 Stress Scales.
}

Juan Luis Delgado-Gallegos ${ }^{1 \#}$, Gerardo R. Padilla-Rivas ${ }^{1 \#}$, Erika Zuñiga-Violante ${ }^{2}$, Gener Avilez-Rodriguez ${ }^{3}$, Daniel Arrellanos-Soto ${ }^{1}$, Hector Franco Villareal ${ }^{4}$, María de los Angeles Cosio-León ${ }^{5}$, Gerardo Salvador Romo-Cardenas $^{3 \$}$, and Jose Francisco Islas ${ }^{1 \$}$

1. Departamento de Bioquímica y Medicina Molecular, Facultad de Medicina, Universidad Autónoma de Nuevo León, Avenida Dr. Eduardo Aguirre Pequeño, Col. Mitras Centro, Monterrey, N.L., México.

2. Universidad de Montemorelos, Av. Libertad 1300 Pte, Matamoros, 67515

3. Facultad de Ingeniería, Arquitectura y Diseño, Universidad Autónoma de Baja California, Carr. Transpeninsular 391, 22860 Ensenada, B.C., México

4. Althian Clinical Research, Calle Capitán Aguilar Sur 669, Col. Obispado Monterrey, N.L., México.

5. Universidad Politécnica de Pachuca, Carretera, Carretera Ciudad Sahagún-Pachuca Km. 20, ExHacienda de Santa Bárbara, 43830 Zempoala, Hidalgo, México.

\# These authors contributed equally to this work.

\$Co-corresponding authors:

Gerardo Salvador Romo-Cardenas romo.gerardo@uabc.edu.mx

Jose Francisco Islas jislas.me0117@uanl.edu.mx

Abstract:

To mitigate the COVID-19 infection, many world governments endorsed the cessation of non-essential activities, such as the school attendance. Thereby, forcing the evolution of the teaching model to the virtual classroom. In the present work we show the application of a modified version of the adapted COVID-19 stress scales (ACSS) which also included teaching anxiety and preparedness, and resilience for academic professionals in Mexico, during the unprecedented transformation of the education system undergone in the COVID-19 quarantine. Most of the studied variables: gender, age, academic degree, household occupants, having a disease, teaching level, teaching mode, work hours, resilience, teaching anxiety and preparedness, and fear of being an asymptomatic patient (FOBAP), showed significant statistical correlation between each other $(p<0.050)$ and to the 6 areas of the ACSS (danger, contamination, social economical, xenophobia, traumatic stress and compulsive checking). Our results further showed that the perceived stress and anxiety, fell into the category of absent to mild with only the danger section of the ACSS falling into the moderate category. Finally, resilience generated throughout the quarantine, seems to be a predictor of the adaptation the academic professional has undergone to cope with stress.

Key words: Adapted COVID-Stress Scales; Stress in Academic Professionals; Resilience to COVID stress in Academia 


\section{Introduction}

As the COVID-19 pandemic continues to grow worldwide, it has strained resources of different countries to the utmost capacity in terms of economic, technical, and human resources, leading to an unprecedented world crisis.

The social contingency derived from the pandemic, has put in perspective many aspects of the daily life. One strategy to mitigate the spreading of the COVID-19, was the cessation of non-essential activities, such as school attendance. Although considered a non-essential activity, education remains vital to the advancement of society $[1,2]$. This served as a precedence to develop strategies to mobilize the academic professional from the classroom setting to a virtual classroom, using diverse electronic platforms e.g., Zoom ${ }^{\circledR}$, Microsoft TEAMS $^{\circledR}$, Google Classroom $^{\circledR}$, Moodle ${ }^{\circledR}$ and others [3]. Additional challenges have also arisen in order to keep professors connected to their pupils, reduce their confusion and stress, and maintain a focus on learning [4]. In developing democracies such as Mexico, internet access posed added difficulties to the academic professional as even-though the country has made great strides, it continues to struggle with affordable country wide access, making it difficult for full integration of the students the virtual classroom $[5,6]$.

In Mexico, over 30 million students and 2 million professionals, use a variety of learning facilities each day [7]. With the emergence of the COVID-19 pandemic, several academic institutions, in conjunction, with the Mexican Ministry of Health on March 14, 2020 issued a series of guidelines in order to prevent and reduce the risk of infection of COVID-19. Out of these guidelines there was the explicit directive, as of March $20^{\text {th }}$, to suspend all in-person school activities, which continues being enforced to this day $[7,8]$. This mandate's purpose serves to maintain afloat the educational system and help to develop it technologically. Because of this new technological requirements, academic professionals had to adapt, thus the need to be up-to-date in diverse technological tools and platforms, hence integrating a combination of traditional resources together with the application of these tools $[9,10]$. However, this directly puts high levels of pressure and stress on academic professionals, particularly for those who rarely use these settings, challenging their ability to use technology and innovation to make students immerse themselves in the learning experience. However, from the academic professional perspective in a recent study by Martinez-Garces et al., at the National Autonomous University of Mexico revealed that amongst the fundamental problems they faced during the contingency with technological advancement were of logistical, and social-affective nature $[3,10]$. Researchers further mentioned that computer information literacy, communication and collaboration, and problem-solving skills are the most developed competencies, whereas digital content creation and security are the weakest [11]. Therefore, it is imperative that academic professional modernize, as in some cases some professionals may not be as familiar with recent technology[3,10,12]. The most underestimated problem during this pandemic continues being work overload that academic professionals endure on a daily basis, which is exacerbated, if they are not prepared because of technology impairdness, additionally there are those who, due to the nature of their work require going at least partially, which implies the continuous use of PPE, and the combination of preparing material at a distance and in person $[2,12,13]$. As a result, this leads to physical exhaustion, emotional fatigue and the fear of infection by contact with students or other teachers or someone in their family becoming infected, which causes even more anxiety and stress [14-16]. This overload of physical, mental and emotional stress can be so significant that it can trigger the development of mild to severe psychiatric disorders such as depression, anxiety, and even burnout syndrome [17-19].

A study by Wang et.al. 2020 showed the impact of the psychological impact at the initial stages of the pandemic, in which more than the half of the population classified as moderate to severe, and a third as moderate to severe. Added to the stress, anxiety, depression, fear, insomnia, xenophobia, eating 
disorders caused by the COVID-19 pandemic. Studies also showed that stress can be beneficial as it helps preserve homeostasis, self-motivation, and survival. Nonetheless, it can produce alterations in memory and cognition, learning, immune response, sleep, cardiovascular health, and endocrine system. Furthermore, an important target of treatment in depression and anxiety is resilience, which can be viewed as a measure of coping ability and thrive in the face of adversity [20]. Additionally, resilience has shown to be a predictor in antidepressant medication [21]. Thus, managing stress is key to keep an adequate mental health.

In our previous studies, we worked on understanding how stress affects the medical professionals; one of the most affected groups during the pandemic, as they are at the frontline dealing directly with the disease $[16,22]$. Now we will focus on evaluating the vulnerability and adaptability of the academic professional in a developing democracy setting, such is the case of Mexico, as they have to face new challenges to the entire academic setting, and even though hope to end the pandemic seem near $[23,24]$, the potential new normal will surely have a high technological component. In this work we used the COVID-Stress Scales originally developed by Taylor el al., and adapted by our group but now to the academic professional $[16,25]$. In addition, we have now further explored adaptation to the pandemic by measuring the resilience developed in the academic professional as they continue treading forward during the pandemic.

\section{Material and Methods}

This study explores a further application of the adapted COVID stress scales (ACSS) modified for the academic professional in Mexico. We based this work on the ACSS by Delgado Gallegos et al., used to evaluate stress in the daily life of medical professionals. Our questionnaire analyzes the six psychometric areas of the ACSS, additionally we studied resilience, Teaching Anxiety and Preparedness, and the fear of being an asymptomatic patient (FOBAP). Additionally, we added sociodemographic questions about gender, age, level of academic studies, geographic region. The full questionnaire is shown in Table 1

Table 1 Adapted COVID STRESS SCALES for Academic professionals in Mexico

Initial Questions

$1 \quad$ Desea usted participar en el cuestionario?

$2 \quad$ Cual es su genero?

$3 \quad$ Cual es su edad?

$4 \quad$ Cual es su grado académico?

$5 \quad$ Estado en donde vide actualmente

$6 \quad$ Cuantas personas viven en su casa, incluyendo usted?

$7 \quad$ Padece usted alguna enfermedad de riesgo?

$8 \quad$ Nivel académico en el que imparte clase

$9 \quad$ En que modalidad imparte clase? 
10

11

En caso de contestar presencial o mixto, en la pregunta anterior

¿Cuántas horas pasa usted en el area física de trabajo a la semana?

Cuantas horas trabaja al dia?

\section{Teaching Preparedness and Anxiety}

12 Se siente preocupado por el uso y manejo de las herramientas tecnológico?

13 Qué tan efectiva ha sido la capacitación que recibido durante el periodo de la contingencia sanitaria?

-14 Siente que tiene el equipo necesario para dar clases de forma virtual?

15 Le preocupa regresar al aula de forma presencial en los próximos meses?

\section{Section 1 (Danger)}

$6 \quad$ Estoy preocupado por contraer el virus

$8 \quad$ Me preocupa que la higiene básica (por ejemplo, el lavado de manos) no sea suficiente para mantenerme a salvo del virus

$9 \quad$ Me preocupa que nuestro sistema de salud no pueda mantenerme a salvo del virus

10 Me preocupa no poder mantener a mi familia a salvo del virus

11 Me preocupa que nuestro sistema de salud no pueda proteger a mis seres queridos

12 Me preocupa que el distanciamiento social no sea suficiente para mantenerme a salvo del virus

\section{Section 2 (Fear of Contamination)}

13 Me preocupa que las personas a mi alrededor me infecten con el virus

Me preocupa que si tocara algo en un espacio público (por ejemplo, pasamanos, manija de la puerta), pueda contraer el virus

15 Me preocupa que si alguien tosiera o estornudara cerca de mí, podría contraer el virus.

16 Me preocupa que pueda contraer el virus al manejar dinero o usar una máquina de tarjeta de débito/crédito

17 Estoy preocupado por hacer transacciones en efectivo

18 Me preocupa que mi paquetería / correo haya sido contaminado durante su tránsito y manejo.

19 Me preocupa convivir con personas recuperadas de COVID-19. 
Me preocupa que las tiendas de comestibles se queden sin comida

Me preocupa que las tiendas de comestibles se queden sin remedios para el resfriado o la gripe

Me preocupa que las farmacias se queden sin medicamentos recetados

Me preocupa que las tiendas de comestibles se queden sin agua

Me preocupa que las tiendas de comestibles se queden sin productos de limpieza o desinfectantes.

Me preocupa que las tiendas de comestibles cierren

Me preocupa perder mi trabajo.

La cuarentena ha afectado la calidad de mi trabajo.

\section{Section 4 (Xenophobia)}

$28 \quad$ Me preocupa que personas fuera del estado estén propagando el virus.

29 Me preocupa que las personas que conozco, que viven fuera de mi estado, puedan tener el virus.

30 Me preocupa entrar en contacto con personas fuera del estado porque pueden tener el virus.

$31 \quad$ Me preocupa que personas extranjeras estén propagando el virus porque no están tan limpios como nosotros

Si fuera a un restaurante especializado en alimentos extranjeros, me preocuparía contraer el virus con el virus.

\section{Section 5 (Traumatic stress)}

$34 \quad$ Tuve problemas para dormir porque me preocupaba el virus

35 Tuve malos sueños sobre el virus

$36 \quad$ Pensé en el virus cuando no quise

37 Aparecieron en mi mente, contra mi voluntad, imágenes mentales inquietantes sobre el virus

$38 \quad$ Tuve problemas para concentrarme porque seguía pensando en el virus

39 Los recordatorios del virus me provocaron reacciones físicas, como sudoración o latidos fuertes del corazón.

Section 6 (Compulsive Checking) 
40

$41 \quad$ Reviso videos de YouTube sobre COVID-19

42 Solicitó tranquilidad a amigos o familiares sobre COVID-19

43 Reviso mi propio cuerpo en busca de signos de infección ( $p$. Ej., Tomando mi temperatura)

44 Pido consejo a los profesionales de la salud (por ejemplo, médicos o farmacéuticos) sobre COVID-19

$45 \quad$ Busco en Internet tratamientos para COVID-19

$46 \quad$ He sido diagnosticado con COVID-19

Fear of Beaing an asymptomatic patient

47 Estoy preocupado de ser asintomático y contagiar a mis seres queridos.

$48 \quad$ Tengo miedo de reinfectarme con COVID-19.

\section{Resilience}

$49 \quad$ En general, me tomo las cosas con calma.

50 Soy una persona con adecuada autoestima.

51 La seguridad en mí mismo, me ayuda a salir de momentos difíciles.

52 En una emergencia soy alguien en quien la gente puede confiar.

53 Cuando estoy en una situación difícil por lo general puedo encontrar una salida.

Final questions for future follow-up

$54 \quad$ ¿Le interesaría en un futuro participar en un cuestionario para seguimiento de su salud mental?
$55 \quad$ Le agradecemos su interés y le pedimos, por favor nos deje una dirección de correo electrónico

The questionnaire was written using MS Forms $®$ (Microsoft Corporation, Redwood, WA, United States), and was applied remotely through a web link. The questionnaire was distributed by electronic means to academic professionals nationwide, through a partnership with the educational system in the private sector, during a one-month period in December 2020. Professionals surveyed ranged from elementary to postgraduate education teaching levels. All academic professional participating acknowledged being $>18$ years and gave consent by electronic means for their inclusion in the study. 
Our questionnaire was developed using a Likert scale system with an increasing point based system [26]. Results of the questionnaire were tallied, and statistical analysis correlations were processed using IBM SPSS Statistics for Windows, version 23.0 (IBM Corp., Armonk, NY, USA) with Pearson's chi-squared ratio of 0.05 .

Results from the questionnaire were classified as described in our previous work [16,27]. Nonetheless, several modifications were done as Teaching Anxiety and Preparedness (4 questions), and Resilience (5 questions) sections were added, additionally questions were added to Contamination (7 questions), Social Economical ( 8 total questions), and FOBAP ( 2 total questions). Results were classified as following: Section with 2 questions Absent $=0-2$, Mild = 3-4, Moderate $=5-6$, Severe $=7-8$. Section with 4 questions Absent $=0-4$, Mild $=5-8$, Moderate $=9-12$, Severe $=13-16$. Sections with 6 questions (original scale) Absent $=0-$ 6 , Mild $=7-12$, Moderate $=13-18$, Severe $=19-24$. Sections with 7 questions Absent $=0-7$, Mild $=8-14$, Moderate $=15-21$, and Severe 22-28. Sections with 8 questions Absent $=0-8$, Mild =9-16, Moderate $=17$ 24, Severe $=25-32$. Finally, resilience section was categorized as: Very low (0-4), Low (5-8), Normal (9-12), High (13-16), and Very high (17-20). In addition, general scale totals were done using cumulative scores for each section.

\section{Results}

From a total of 223 participants recruited, three declined to take part in the study. Therefore, our results for the study were of a total of 220 participants. We should note that participants were not required to answer all sections to advance through the questionnaire. The general sociodemographic information for all consenting participants is presented in Table 2.

Noticeably, 74 (33.78\%) participants were in the age range of 31-40 years; the most frequent range, additionally 6 (2.71\%) had a high school degree, 155 (70.13\%) participants had a college degree, and 60 (27.14\%) had a graduate degree. Moreover, participants living occupancy was for 4 occupants $27.39 \%$ (n $=60)$; the most frequent answer, while $36.15 \%(n=77)$ of participants answered working $>8$ hours per day and $84.47 \%$ taught online, only $2.73 \%$ reported in-person teaching and $7.3 \%$ reported mix mode teaching. Our results also showed a gender gap as there is a 3:1 ratio of Females to Males with the majority of participants teaching at basic levels such as Elementary (34.86\%), Junior high (24.34\%) and High school $(22.36 \%)$, while at professional $16.44 \%$ and graduate $1.97 \%$. Finally, as comorbidities have been reported to exacerbate COVID-19[28,29], we further did frequency counts. Over $60 \%$ of responders mention no important comorbidities, yet $12 \%$ mentioned having at least obesity and $7 \%$ diabetes. Moreover, cardiac diseases were seen present in 7\% of the participants and Pulmonary diseases in 3\%. Figure 1 shows frequency counts for social demographic variables and percentage distributions. 
Table 2 Social demographic profile of participants $(n=220)$

\begin{tabular}{|c|c|c|c|c|}
\hline Male & 28.5 & & & \\
\hline Female & 71.04 & & & \\
\hline Others & 0.45 & & & \\
\hline \multicolumn{5}{|c|}{ Academic degree } \\
\hline Highschool & 2.71 & & & \\
\hline Bachelor & 70.13 & & & \\
\hline Graduate & 27.14 & & & \\
\hline $\begin{array}{l}\text { Household } \\
\text { occupants }\end{array}$ & $27.39 *$ & 1 & $>4$ & 4 \\
\hline Work hours & $36.15 *$ & 8 & $>8$ & $>8$ \\
\hline \multicolumn{5}{|l|}{ Teaching mode } \\
\hline Presential & 2.73 & & & \\
\hline Online & 84.47 & & & \\
\hline Mix & 7.3 & & & \\
\hline Does not apply & 5.47 & & & \\
\hline \multicolumn{5}{|l|}{ Teaching level } \\
\hline Elementary & 34.86 & & & \\
\hline Junior high & 24.34 & & & \\
\hline Highschool & 22.36 & & & \\
\hline Bachelor & 16.44 & & & \\
\hline Graduate & 1.97 & & & \\
\hline \multicolumn{5}{|l|}{ Diseases } \\
\hline Diabetes & 7 & & & \\
\hline Cardiac & 7 & & & \\
\hline Pulmonary & 3 & & & \\
\hline Autoinmune & 2 & & & \\
\hline Obesity & 12 & & & \\
\hline Cancer & 1 & & & \\
\hline HIV & $<1$ & & & \\
\hline Others & 8 & & & \\
\hline None & 60 & & & \\
\hline
\end{tabular}

$*$ = percentage of the mode 
Figure 1 Frequency count bar graphs for A) Age, B) Academic degree, C) Teaching mode, D) Household Occupants, E) Work hours. Percentage distribution for F) Teaching level, G) Gender, H) Diseases

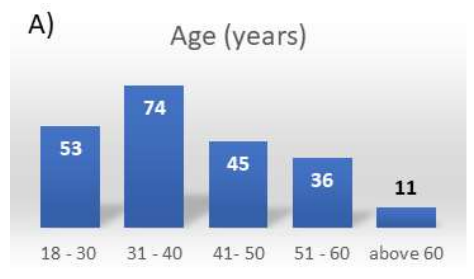

D) Household Occupants

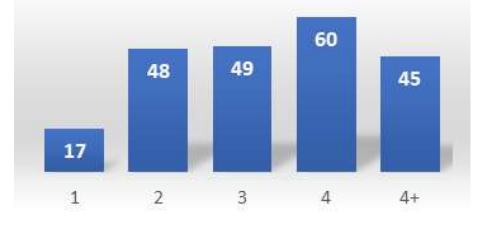

F)

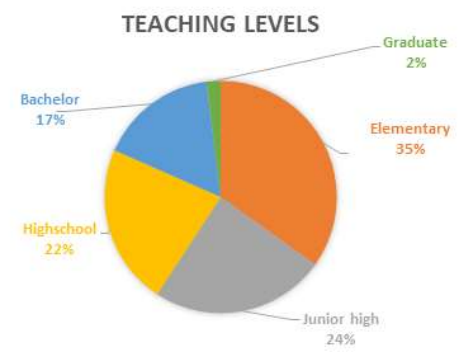

B) Academic Degree

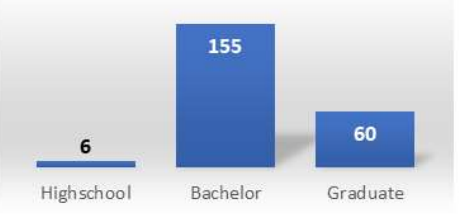

E)

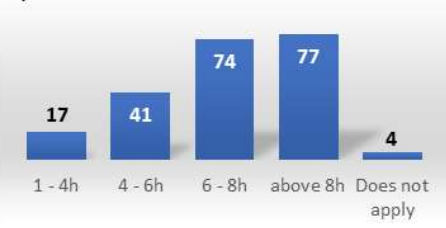

G)

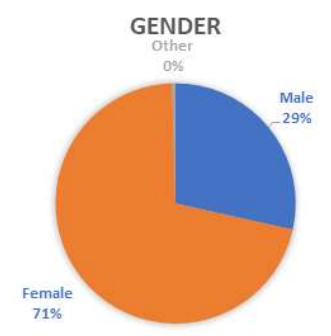

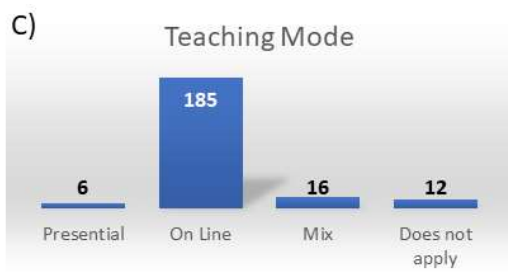$$
\text { (1) }
$$

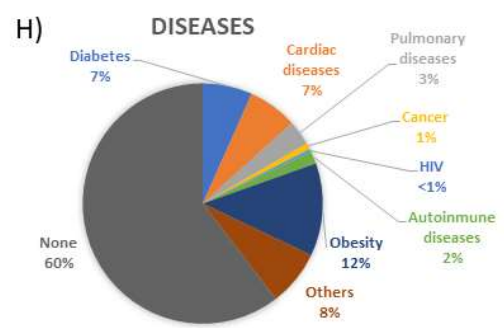

Next, we evaluated stress frequency as interpreted by the ACSS for the six sections to understand into which classification do the majority of academic professionals fall into. Our results for the most frequent classification in ACSS areas showed: Danger (Moderate 42.3\%, $n=93$ ), Contamination (Mild 47.7\%, $n=105$ ), Xenophobia (Mild 41.8\%, n=92), Social Economical (Mild 34\%, $n=74$ ), Traumatic Stress (Absent $65.5 \%$, $\mathrm{n}=144$ ), Compulsive Checking (Absent 43.2\%, $n=95$ ), and for the Total sections (Mild 50\%, $n=110$ ). Additionally, we further tested for Teacher Anxiety and Preparedness (Mild 63.6\%, $n=140$ ) and FOBAP (Absent $40.9 \%, n=90$ ). We did one final count in order to understand resilience, for which result showed most academic professionals had very high resilience $(56.2 \%, n=118)$. Table 3 shows the full COVID related stress frequency and classification, and Figure 2 graph percentages for most representative frequencies. 
Table 3 COVID-related stress frequency for the ACSS, Teaching Anxiety and Preparedness, and Resilience for Academic Professionals

\begin{tabular}{|c|c|c|c|c|c|c|c|c|}
\hline & \multicolumn{2}{|c|}{ Danger } & \multicolumn{2}{|c|}{ Contamination } & \multicolumn{2}{|c|}{ Xenophobia } & \multicolumn{2}{|c|}{ Social Economical } \\
\hline & $\mathrm{N}$ & $\%$ & $\mathrm{~N}$ & $\%$ & $\mathrm{~N}$ & $\%$ & $\mathrm{~N}$ & $\%$ \\
\hline ABSENT & 21 & 9.5 & 46 & 20.9 & 47 & 21.4 & 38 & 17.5 \\
\hline MILD & 70 & 31.8 & 105 & 47.7 & 92 & 41.8 & 74 & 34.0 \\
\hline MODERATE & 93 & 42.3 & 59 & 26.8 & 66 & 30.0 & 72 & 33.1 \\
\hline SEVERE & 36 & 16.4 & 10 & 4.5 & 15 & 6.8 & 33 & 15.4 \\
\hline \multirow[t]{3}{*}{ Total } & & 100.0 & & 100.0 & & 100.0 & & 100.0 \\
\hline & \multicolumn{2}{|c|}{ Traumatic Stress } & \multicolumn{2}{|c|}{ Compulsive Cheking } & \multicolumn{2}{|c|}{ Total Sections } & & \\
\hline & $\mathrm{N}$ & $\%$ & $\mathrm{~N}$ & $\%$ & $\mathrm{~N}$ & $\%$ & & \\
\hline ABSENT & 144 & 65.5 & 95 & 43.2 & 37 & 16.9 & & \\
\hline MILD & 49 & 22.3 & 82 & 37.3 & 110 & 50.0 & & \\
\hline MODERATE & 18 & 8.2 & 32 & 14.5 & 65 & 29.5 & & \\
\hline SEVERE & 9 & 4.1 & 11 & 5.0 & 8 & 3.6 & & \\
\hline \multirow[t]{3}{*}{ Total } & & 100.0 & & 100.0 & & 100.0 & & \\
\hline & \multicolumn{2}{|c|}{$\begin{array}{l}\text { Teaching Anxiety and } \\
\text { Prepardness }\end{array}$} & \multicolumn{2}{|c|}{ FOBAP } & & & & \\
\hline & $\mathrm{N}$ & $\%$ & $\mathrm{~N}$ & $\%$ & & & & \\
\hline ABSENT & 14 & 6.4 & 90 & 40.9 & & & & \\
\hline MLLD & 140 & 63.6 & 76 & 34.5 & & & & \\
\hline MODERATE & 65 & 29.5 & 40 & 18.2 & & & & \\
\hline SEVERE & 1 & 0.5 & 14 & 6.4 & & & & \\
\hline \multirow[t]{3}{*}{ Total } & & 100.0 & & 100.0 & & & & \\
\hline & \multicolumn{2}{|c|}{ Resilience } & & & & & & \\
\hline & $\mathrm{N}$ & $\%$ & & & & & & \\
\hline Very Low & 10 & 4.7 & & & & & & \\
\hline Low & 4 & 2.0 & & & & & & \\
\hline Normal & 21 & 9.9 & & & & & & \\
\hline High & 67 & 31.9 & & & & & & \\
\hline Very High & 118 & 56.2 & & & & & & \\
\hline Total & & 100.0 & & & & & & \\
\hline
\end{tabular}




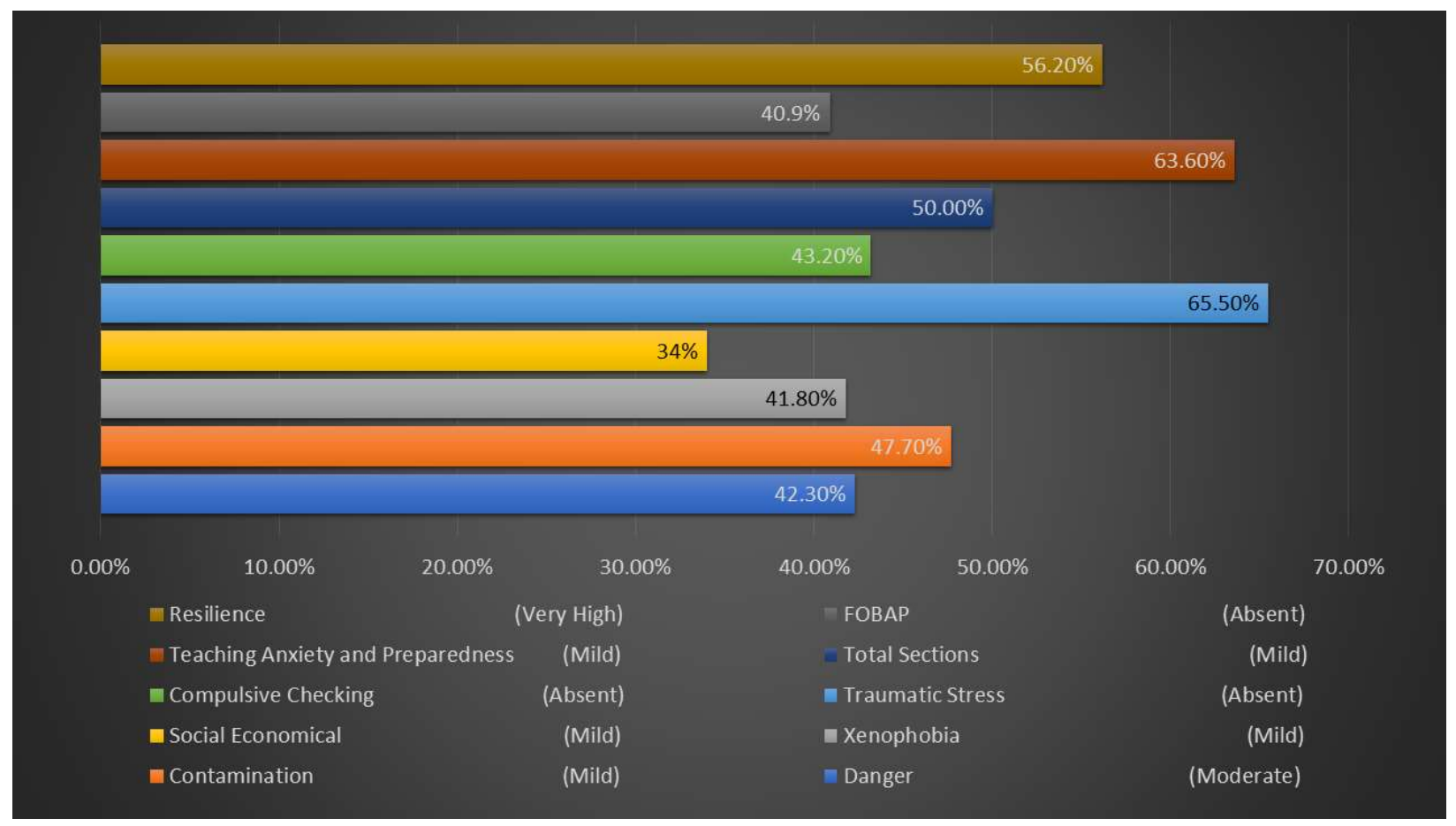

Figure 2 Most representative COVID-related stress frequency (shown as percentage) for the ACSS, Teaching Anxiety and Preparedness, and Resilience for Academic Professionals.

Since our primary goal was to analyze how the COVID-19 has affected academic professionals, we analyzed the statistical correlation between all survey variables. All correlations can be seen on Table 4 . Females showed correlations $(p<0.001)$ to all variables (Resilience, Teaching Anxiety and Preparedness, FOBAP, Danger, Contamination, Social Economical, Xenophobia, Traumatic stress, Compulsive checking, Total Sections), meanwhile males did not show statistical correlation to Social Economical $(p<0.076)$ (Supplemental Table 1). Age, 18 to 30 y showed correlation to all variables except Social Economical ( $p<$ 0.298), 31 to 40 y and 41 to 50 y showed statistical correlation to all variables, 51 to 60 showed correlation to all variables except Social Economical $(p<0.774)$ and Contamination $(p<0.076)$, finally participants above 60 y only showed correlations to Resilience $(p<0.029)$, Teaching Anxiety and Preparedness $(p<$ 0.004 ), and FOBAP ( $p$ 0.035) (Supplemental Table 2). Academic degree, participants with only a high school degree did not show any correlations, for those with a professional degree and above statistical correlations were seen in all variables (Supplemental Table 3). For Household occupants, 1-person show statistical relevance with FOBAP $(p<0.010)$, Contamination $(p<0.039)$, and Traumatic stress $(p<0.007)$, for those living with an additional companion statistical correlation were seen in all variables except for Social Economical $(p<0.771)$. Interestingly, individuals living in households of a total of 3 or 4 resulted in statistical correlation to all variables, yet individual living with 4 or more companions the variables of FOBAP $(p<0.066)$ and Social Economical $(p<0.057)$ were not statistically relevant (Supplemental Table 4). Expectantly, participants with a comorbidity showed statistical relevance to all variables (Supplemental Table 5). Academic professionals teaching at an Elementary, Junior High School, High School, and Bachelor levels showed statistical relevance to all variables, meanwhile those teaching at a Graduate level did not show any correlations (Supplemental Table 6). Moreover, for those teaching Inperson no correlation to any variable was seen, whereas professionals teaching online statistical relevance was seen in all variables, strikingly, professionals teaching in a mix form show correlation to Teaching Anxiety and Preparedness ( $<<0.039)$, Traumatic stress $(p<0.001)$, Compulsive checking, and Total sections $(p<0.002)$ (Supplemental Table 7). For those professionals working for up to $4 \mathrm{~h}$, results showed 
statistical relevance only to Resilience $(p<0.047)$, Teaching Anxiety and Preparedness $(p<0.023)$, and both Danger and Compulsive checking $(p<0.028)$. For those professionals working from 4 to $6 h$, results were relevant to all variables except in the case of Social Economical $(p<0.191)$. Lastly, for those professionals working 6 or more hours all variables presented statistical correlations (Supplemental Table 8).

Finally, we analyzed Resilience, Teaching Anxiety and Preparedness, and FOBAP between themselves and the ACSS. As expected, Resilience showed statistical correlation to the ACSS, yet not to either Teaching Anxiety and Preparedness $(p<0.631)$ or FOBAP $(p<0.185)$ (Supplemental Table 9). Teaching Anxiety and Preparedness showed statistical relevance to Contamination $(p<0.001)$, Social Economical $(p<0.007)$, Xenophobia $(p<0.003)$, and Total Sections $(p<0.001)$ (Supplemental Table 10). Lastly, FOBAP, showed correlations to the whole ACSS and Total Sections (Supplemental Table 11).

Table 4 Statistical correlations of all variables: Resiliance, Teaching Anxeity and Preparednes, FOBAP, Sociodemographic profiles, ACSS and Total Sections.

\begin{tabular}{|c|c|c|c|c|c|c|c|c|c|c|}
\hline & Resilience & $\begin{array}{l}\text { Teaching anxiety } \\
\text { and preparedness }\end{array}$ & FOBAP & Danger & Contamination & $\begin{array}{c}\text { Social } \\
\text { Economical }\end{array}$ & Xenophobia & $\begin{array}{c}\text { Traumatic } \\
\text { Stress }\end{array}$ & $\begin{array}{c}\text { Compulsive } \\
\text { checking }\end{array}$ & Total Sections \\
\hline \multicolumn{11}{|l|}{ Gender } \\
\hline Females & $p<0.001$ & $p<0.001$ & $p<0.001$ & $p<0.001$ & $p<0.001$ & $p<0.001$ & $p<0.001$ & $p<0.001$ & $p<0.001$ & $p<0.001$ \\
\hline Males & $p<0.001$ & $p<0.001$ & $p<0.001$ & $p<0.001$ & $p<0.001$ & $p<0.076$ & $p<0.002$ & $p<0.001$ & $p<0.001$ & $p<0.001$ \\
\hline \multicolumn{11}{|l|}{ AGE } \\
\hline 18 to $30 y$ & $p<0.001$ & $p<0.001$ & $p<0.001$ & $p<0.010$ & $p<0.001$ & $p<0.298$ & $p<0.001$ & $p<0.001$ & $p<0.001$ & $p<0.001$ \\
\hline 31 to $40 y$ & $\mathrm{p}<0.001$ & $p<0.001$ & $p<0.001$ & $p<0.003$ & $\mathrm{p}<0.001$ & $p<0.001$ & $p<0.001$ & $p<0.001$ & $p<0.001$ & $p<0.001$ \\
\hline 41 to $50 y$ & $p<0.001$ & $p<0.001$ & $p<0.004$ & $p<0.001$ & $\mathrm{p}<0.001$ & $p<0.036$ & $p<0.001$ & $p<0.001$ & $p<0.031$ & $p<0.001$ \\
\hline 51 to $60 y$ & $p<0.002$ & $p<0.001$ & $p<0.001$ & $p<0.042$ & $p<0.076$ & $p<0.774$ & $p<0.042$ & $p<0.001$ & $p<0.003$ & $p<0.001$ \\
\hline Above $60 y$ & $p<0.029$ & $p<0.004$ & $p<0.035$ & $p<0.234$ & $\mathrm{p}<0.484$ & $p<0.631$ & $\mathrm{p}<0.200$ & $p<0.178$ & $p<0.147$ & $p<0.529$ \\
\hline \multicolumn{11}{|l|}{ Academic degree } \\
\hline Highschool & $\mathrm{p}<0.655$ & $p<0.819$ & $p<0.819$ & $p<0.449$ & $p<0.449$ & $p<0.449$ & $p<0.655$ & $p<0.655$ & $p<0.449$ & $p<0.819$ \\
\hline Bachelor & $p<0.001$ & $p<0.001$ & $p<0.001$ & $p<0.001$ & $p<0.001$ & $p<0.001$ & $p<0.001$ & $p<0.001$ & $p<0.001$ & $p<0.001$ \\
\hline Graduate & $p<0.001$ & $p<0.001$ & $p<0.001$ & $p<0.001$ & $\mathrm{p}<0.001$ & $p<0.022$ & $p<0.001$ & $p<0.001$ & $p<0.001$ & $p<0.001$ \\
\hline \multicolumn{11}{|l|}{ Household occupants } \\
\hline 1 & $p<0.099$ & p 0.090 & $p<0.010$ & $p<0.172$ & $p<0.039$ & $p<0.112$ & $p<0.444$ & $p<0.007$ & $p<0.174$ & $p<0.144$ \\
\hline 2 & $p<0.001$ & $p<0.001$ & $p<0.001$ & $p<0.029$ & $p<0.001$ & $p<0.771$ & $p<0.037$ & $p<0.001$ & $p<0.001$ & $p<0.019$ \\
\hline 3 & $p<0.001$ & $p<0.001$ & $p<0.001$ & $p<0.001$ & $p<0.001$ & $p<0.050$ & $p<0.001$ & $p<0.001$ & $p<0.001$ & $p<0.001$ \\
\hline 4 & $p<0.001$ & $p<0.001$ & $p<0.002$ & $p<0.001$ & $p<0.001$ & $p<0.001$ & $p<0.008$ & $p<0.001$ & $p<0.001$ & $p<0.001$ \\
\hline more than 4 & $p<0.001$ & $p<0.001$ & $p<0.066$ & $p<0.017$ & $p<0.017$ & $p<0.057$ & $p<0.001$ & $\mathrm{p}<0.001$ & $p<0.002$ & $p<0.001$ \\
\hline \multicolumn{11}{|l|}{ Diseases } \\
\hline w/ comorbidity & $p<0.001$ & $p<0.001$ & $p<0.001$ & $p<0.001$ & $p<0.001$ & $p<0.003$ & $p<0.001$ & $p<0.001$ & $p<0.002$ & $p<0.001$ \\
\hline \multicolumn{11}{|l|}{ Teaching levels } \\
\hline Elementary & $p<0.001$ & $p<0.001$ & $p<0.001$ & $p<0.001$ & $p<0.001$ & $p<0.019$ & $p<0.001$ & $p<0.001$ & $p<0.001$ & $p<0.001$ \\
\hline Junior Highschool & $p<0.001$ & $p<0.001$ & $p<0.001$ & $p<0.001$ & $p<0.001$ & $p<0.002$ & $p<0.001$ & $p<0.001$ & $p<0.001$ & $p<0.001$ \\
\hline Highschool & $p<0.001$ & $p<0.001$ & $p<0.001$ & $p<0.001$ & $p<0.001$ & $p<0.050$ & $p<0.001$ & $p<0.001$ & $p<0.001$ & $p<0.001$ \\
\hline Bachelor & $p<0.001$ & $p<0.001$ & $p<0.001$ & $p<0.029$ & $p<0.001$ & $p<0.084$ & $p<0.001$ & $p<0.001$ & $p<0.001$ & $p<0.001$ \\
\hline Graduate & $p<1.000$ & $p<0.414$ & $p<1.000$ & $p<0.607$ & $\mathrm{p}<0.102$ & $p<0.607$ & $p<0.607$ & $p<0.223$ & $p<0.223$ & $p<0.223$ \\
\hline \multicolumn{11}{|l|}{ Teaching mode } \\
\hline In-person & $p<0.100$ & $p<0.607$ & $p<0.414$ & $p<0.607$ & $p<0.607$ & $p<0.607$ & $p<0.223$ & $p<0.607$ & $p<0.607$ & $p<0.607$ \\
\hline Online & $p<0.001$ & $p<0.001$ & $p<0.001$ & $p<0.001$ & $p<0.001$ & $p<0.001$ & $p<0.001$ & $p<0.001$ & $p<0.001$ & $p<0.001$ \\
\hline Mix & $p<0.305$ & $p<0.039$ & $p<0.090$ & $p<0.058$ & $p<0.058$ & $p<0.090$ & $p<0.321$ & $p<0.001$ & $p<0.029$ & $p<0.002$ \\
\hline \multicolumn{11}{|l|}{ Work hours } \\
\hline 0 to $4 \mathrm{~h}$ & $p<0.047$ & $p<0.023$ & $p<0.052$ & $p<0.028$ & $p<0.193$ & $p<0.098$ & $p<0.181$ & $p<0.467$ & $p<0.028$ & $p<0.161$ \\
\hline 4 to $6 \mathrm{~h}$ & $p<0.001$ & $p<0.001$ & $p<0.012$ & $p<0.001$ & $\mathrm{p}<0.002$ & $p<0.191$ & $p<0.001$ & $p<0.001$ & $p<0.001$ & $p<0.012$ \\
\hline 6 to $8 \mathrm{~h}$ & $p<0.001$ & $p<0.001$ & $p<0.001$ & $p<0.001$ & $p<0.001$ & $p<0.010$ & $p<0.001$ & $p<0.001$ & $p<0.001$ & $p<0.001$ \\
\hline more than $8 \mathrm{~h}$ & $p<0.001$ & $\mathrm{p}<0.001$ & $p<0.001$ & $p<0.001$ & $p<0.001$ & $p<0.001$ & $p<0.001$ & $p<0.001$ & $p<0.001$ & $p<0.001$ \\
\hline Resilience & & $p<0.631$ & $p<0.185$ & $p<0.001$ & $p<0.004$ & $p<0.021$ & $p<0.002$ & $p<0.007$ & $p<0.020$ & $p<0.001$ \\
\hline $\begin{array}{l}\text { Teaching anxeity and } \\
\text { preparedness }\end{array}$ & $p<0.631$ & & $p<0.356$ & $p<0.001$ & $p<0.100$ & $p<0.007$ & $p<0.003$ & $p<0.491$ & $p<0.175$ & $p<0.001$ \\
\hline FOBAP & $p<0.185$ & $p<0.356$ & & $p<0.001$ & $p<0.001$ & $p<0.001$ & $p<0.001$ & $p<0.001$ & $p<0.001$ & $p<0.006$ \\
\hline
\end{tabular}

\section{Discussion}

Our general data (Table 3 and Figure 2) indicated that the most common categories in which academic professionals fall into was Absent for Compulsive checking, Traumatic Stress and FOBAP. Falling into the absent category or a normalization to daily activities is primarily the ability of human adaption to stressful environments [30-33]. Albeit not an easy task, as more information has become available, better practices such as social distancing, the use of face masks, and remote communications (no need to be in front of 
students) have completely change the landscape of every day social interactions. As there are less direct interactions, people have normalized the use protective equipment and there is a higher perceived sense of self protection thereby reducing overall stress[34,35]. It is important to note that this perceived sense of self protection is unfortunately a false sense of security as clearly the levels of infection continue at a rise[36]. In the CDC's report for January 13, 2021, researchers estimate anywhere between 1,300,000 to 2,400,000 new cases by February 6, 2021 in the US alone [37]. Nevertheless, in the advent of potential treatments such as vaccines there continues to be hope at the end of the tunnel.

The nature of academics is to prepare young minds for the challenges of today and for the world of tomorrow. Interestingly, our results show that Teaching Anxiety and Preparedness, Social Economical, Contamination, Xenophobia and Total sections resulted in generally Mild. Statistically, Teaching Anxiety and Preparedness, as an individual area correlated with having an academic degree; no surprise as being better prepared should be an indicator of less stress, and with all sociodemographic variables, meaning that even though online teaching has continued now for 2 periods (semesters) stress continues, particularly as professionals continue to struggle to have students fully engaged in their activities[1,38,39]. It should be noted, that the Social Economical stress indicator did not correlate with males, nor with the youngest or oldest professionals. Strikingly, it was highly relevant to having a comorbidity (potential to exacerbate by COVID-19), working with young (non-professional level) students and at least working $6 \mathrm{~h}$ per day. As mentioned earlier, the herculean task is not only to teach young minds, but to maintain them engage. Unlike professional level students (or Graduates) were recording lectures is an advantage, younger minds are more prone to household distractors. In Mexico, a typical household of 4 there are at least 2 students who may have to share internet time or educational TV, which in it-self becomes a distractor [6], thereby overloading the academic professional's task. Hence, it is no surprise to see the correlation of those working for 6 or more hours as strategies to put forth this mention effort take up much of their time. Meanwhile the areas of Contamination and Xenophobia, as the fear of people from outside the state of residence coming and spreading the disease resulted in a high frequency of strongly agreed. From our frequency data, danger was the only area showing Moderate, we should note that no category showed severe. Finally, the most common frequency for Resilience was Very High.

From the results of the recollected sociodemographic profile data as seen on Table 2 and the statistical relevance as shown in Table 4, there seems to be high correlation between gender and the studied variables, except in the social economical section of the ACSS, where the females showed a higher concern than males (ref). Interestingly, in age the elderly $>50$ years, seem to have lower anxiety and stress related to COVID-19, with high levels of resilience. Could resilience be developed over a lifetime? Or is the ageold phrase "wisdom comes with age" hold true, particularly for academic professionals. Recently, Pearman et al., denoted that proactive coping was a protective measure against COVID-19 related stress. As older patients are more prone to have complications if infected, hence they have a more hands-on attitude towards complying with restrictive measures and even developing positive habits based on earlier experience[40].

As stated earlier, having a college degree seems to be a determining factor correlating with all variables. Much like experience education plays a role in mental well-being, although at a personal level social anxiety can induce stress, education ensures preparedness a minimum requirement in today's ever more challenging economy. Particularly, we stand in an environment where technology is key, as COVID19 continues, for academic professionals we have seen that distance educating is the norm, and that challenges arise with young students for engagement, hence it is no surprise that with better preparation, professional's strive becoming less stressed $[1,10]$. 
Another interesting variable survey was the amount of household occupants. Understandably, participants with 2 or more household occupants seem to develop more stress than 1 occupant, which can be explained due to the preoccupation of getting sick through a companion or even getting infected from one another. Research has even shown that other factors such as food waste in time of stress is exacerbated by the number of occupants, inevitably putting more strain on the household as a unit[41]. Expectantly, all of the participants with at least 1 comorbidity, showed a statistical correlation to all the studied variables, which can be understood given that they can be more susceptible to developing a severe presentation of COVID-19 [28,29].

In our study, academic professionals taught mostly (>80\%) at a young non-professional level elementary school, junior high school, high school, additionally another $>16 \%$ taught a bachelor level with just less than $2 \%$ teaching at a graduate level. Interestingly, all but the latter had strong correlation to all studied variables. Given that the graduate students do not need as much tutoring as a kid or teenager, graduate professors do not develop as much stress. Because of the transformation of the in-person teaching, to a virtual classroom, the most affected participants were the ones having online teaching, due to the growing demands of the students as well as schoolwork $[1,3,42]$. Understandably, professionals working more hours $(+6 \mathrm{~h})$ tend to develop more stress, than the people working 0 to 4 hours a day, this as a function of strained activity. Resilience, Teaching anxiety and preparedness and FOBAP, have significant correlation to the ACSS, which is understandable, given that they are closely related to the anxiety and stress mechanisms.

In comparison to our previous study where we analyzed healthcare professionals attending COVID19 , results showed that most cases presented mild to moderate stress with traumatic stress, compulsive checking and xenophobia being the most affected areas, along with FOBAP. this might be related to the fact that the healthcare professionals nowadays seem to have endless shifts with a high demand on patient attention, and tend minimize their own malaise when they perceive a higher malaise in their patients [16]. The present study focuses in an academic professional population, our results demonstrated that stress is reduced as many categories resulted in absent to mild, while only the Danger category presented moderate. Additionally, in this study we further analyzed resilience, which demonstrated to be very high for academic professionals, giving an interesting perspective in how two distinct populations of professionals manage stress and anxiety.

\section{Conclusions}

In the present work we have shown the application of the ACSS for academic professionals during the unprecedented transformation of the education system undergone in the COVID-19 quarantine, which has made a significant impact on the work routine on the vast majority of academic professionals. Most of the variables studied: gender, age, academic degree, household occupants, having a disease, teaching level, teaching mode, work hours, resilience, teaching anxiety and preparedness, and FOBAP show significant statistical correlation, to each other and the 6 areas of the ACSS (danger, contamination, social economical, xenophobia, traumatic stress and compulsive checking) which can be translated into a development of mild to moderate perceived stress and anxiety in the daily life, caused by COVID-19.

Although, the high relevance of COVID-19 induced stress and anxiety, the resilience generated during the process, can be a predictor of the adaptation the academic professional has undergone during the quarantine to cope with stress. Although the " $n$ " seems limited, it shows the behavior of a specific population. Understandably, some participants might be unwilling to take part in the survey, due to the 
ever-growing demand of students and schoolwork. Nonetheless, a study with more professionals Is needed to address the rising mental health pandemic due to COVID-19.

Ethical statement:

All participants acknowledged being $>18$ years and gave consent by electronic means for their inclusion in the study. The study was conducted in accordance with the Declaration of Helsinki, and the protocol was approved by the Ethics Committee of Hospital La Misión, Monterrey NL. México. Protocol \#PSY-CEEC-ESP-01.

\section{Supplemental Materials}

Supplemental Table 1. Statistical Correlations for Gender (by Females and Males) vs Adapted COVID STRESS SCALES, Teaching Anxiety and Preparedness, Resilience, and FOBAP. Supplemental Table 2. Statistical Correlations for Age (by groups) vs Adapted COVID STRESS SCALES, Resilience, Teaching Anxiety and Preparedness, and FOBAP. Supplemental Table 3. Statistical Correlations for Academic degree held (High School, Bachelor, Graduate) vs Adapted COVID STRESS SCALES, Teaching Anxiety and Preparedness, Resilience, and FOBAP. Supplemental Table 4. Statistical Correlations for the number of Household occupants vs Adapted COVID STRESS SCALES, Teaching Anxiety and Preparedness, Resilience, and FOBAP. Supplemental Table 5. Statistical Correlations of having a Disease (comorbidity) vs Adapted COVID STRESS SCALES, Teaching Anxiety and Preparedness, Resilience, and FOBAP. Supplemental Table 6. Statistical Correlations of academic levels (Teaching levels) taught vs Adapted COVID STRESS SCALES, Teaching Anxiety and Preparedness, Resilience, and FOBAP. Supplemental Table 7. Statistical Correlations for Teaching mode (in-person, online, and mix) vs Adapted COVID STRESS SCALES, Teaching Anxiety and Preparedness, Resilience, and FOBAP. Supplemental Table 8. Statistical Correlations for Work hours (by range) vs Adapted COVID STRESS SCALES, Teaching Anxiety and Preparedness, Resilience, and FOBAP. Supplemental Table 9. Statistical Correlations for Resilience vs Adapted COVID STRESS SCALES, Teaching Anxiety and Preparedness, and FOBAP. Supplemental Table 10. Statistical Correlations for Teaching Anxiety and Preparedness vs Adapted COVID STRESS SCALES, and FOBAP. Supplemental Table 11. Statistical Correlations for FOBAP vs Adapted COVID STRESS SCALES.

Author Contributions

Research and writing: JLD-G, JFI; Statistical analysis: GRP-R, EZ-V; Conceptualization GAR, DA-S; Resources: HF-V, MdAC-L; Supervision: GSR-C, JFI

Funding

This research received no external funding.

Acknowledgments

Mutual collaboration of all involved institutions.

Conflicts of Interest

The authors declare no conflict of interest 


\section{References}

1. Jandrić, Petar, H.; David, T.; Ian, L.; Paul, M.; Peter, R.; Thomas, M.; D, L.; Allen, Q.; Stewart, P.A.; Carr, P.R.; et al. Teaching in the Age of Covid-19. Postdigital Sci. Educ. 2020, 2, 1069-1230, doi:10.1007/s42439-020-00169-6.

2. Gamage, K.A.A.; Wijesuriya, D.I.; Ekanayake, S.Y.; Rennie, A.E.W.; Lambert, C.G.; Gunawardhana, N. Online delivery of teaching and laboratory practices: Continuity of university programmes during COVID-19 pandemic. Educ. Sci. 2020, 10, 1-9.

3. Sánchez, M.; Martínez, A.; Torres, R.; de Agüero, M.; Hernández, A.; Benavides, M.; Rendón, V.; Jaimes, C. Retos educativos durante la pandemia de COVID-19: una encuesta a profesores de la UNAM. Rev. Digit. Univ. 2020, 21, 1-24.

4. UNESCO Adverse consequences of school closures Available online: https://en.unesco.org/covid19/educationresponse/consequences (accessed on Dec 12, 2020).

5. Ponce-Diaz, R.; Azael-Hernadez, H.; Díaz-Miranda, A.; Díaz-GaonaR The Challenges of Online Education for Public Schools in Mexico. Obs. Educ. Innov. 2020.

6. Press, A. Mexico starts school year using TV, radio and the Internet. Washington Post 2020.

7. Mérida Martínez, Y.; Acuña Gamboa, L.A. Covid-19, Pobreza y Educación en Chiapas: Análisis a los Programas Educativos Emergentes. Rev. Int. Educ. para la Justicia Soc. 2020, 9, 61-82, doi:10.15366/riejs2020.9.3.004.

8. Barajas-Ochoa, A.; NAdrade-Romo, J.S.; Ramos-Santillan, V.O. Challenges for medical education in Mexico in the time of COVID-19. Gac. Med. Mex. 2020, 156.

9. Machado, C.V. Health Policies in Argentina, Brazil and Mexico: Different Paths, Many Challenges. Cien Saude Colet 2018, 22, doi:10.1590/1413-81232018237.08362018.

10. Baptista Lucio, P.; Almazán Zimerman UNETE, A.; Alberto Loeza Altamirano UNETE, C.; cloezaa, M.; Víctor Alfonso López Alcaraz UNETE, uneteorg; Luis Cárdenas Domínguez UNETE, J. Encuesta nacional a docentes ante el Covid-19. Retos para la educación a distancia National Survey to Teachers Facing Covid-19. Challenges for Distance Education. Rev. Latinoam. Estud. Educ. 2020, 50, 41-88.

11. Martínez-Garcés, J.; Garcés-Fuenmayor, J. Competencias digitales docentes y el reto de la educación virtual derivado de la covid-19. Educ. y Humanismo 2020, 22, 1-16, doi:10.17081/eduhum.22.39.4114.

12. Pozo-Rico, T.; Gilar-Corbí, R.; Izquierdo, A.; Castejón, J.L. Teacher training can make a difference: tools to overcome the impact of COVID-19 on primary schools. An experimental study. Int. J. Environ. Res. Public Health 2020, 17, 1-23, doi:10.3390/ijerph17228633.

13. Cordova-Villalobos, J.A.; Macias, A.E.; Hernandez-Avila, M.; Dominguez-Cherit, G.; Lopez-Gatell, H.; Alpuche-Aranda, C.; de León-Rosales, S.P. The 2009 pandemic in mexico: Experience and lessons regarding national preparedness policies for seasonal and epidemic influenza. Gac. Med. Mex. 2017, 153, 102-110.

14. Shah, K.; Chaudhari, G.; Kamrai, D.; Lail, A.; Patel, R.S. How Essential Is to Focus on Physician's Health and Burnout in Coronavirus (COVID-19) Pandemic? Cureus 2020, 12, 10-12, doi:10.7759/cureus.7538.

15. Rajkumar, R.P. COVID-19 and mental health: A review of the existing literature. Asian J. Psychiatr. 2020, 52, 102066.

16. Delgado-Gallegos, J.L.; Montemayor-Garza, R.J.; Padilla-Rivas, G.R.; Franco-Villareal, H.; Islas, J.F. Prevalence of stress in healthcare professionals during the covid-19 pandemic in Northeast Mexico: A remote, fast survey evaluation, using an adapted covid-19 stress scales. Int. J. Environ. Res. Public Health 2020, 17, doi:10.3390/ijerph17207624.

17. Blake, H.; Bermingham, F.; Johnson, G.; Tabner, A. Mitigating the psychological impact of covid- 
19 on healthcare workers: A digital learning package. Int. J. Environ. Res. Public Health 2020, 17, doi:10.3390/ijerph17092997.

18. Moukarzel, A.; Michelet, P.; Durand, A.C.; Sebbane, M.; Bourgeois, S.; Markarian, T.; Bompard, C.; Gentile, S. Burnout syndrome among emergency department staff: Prevalence and associated factors. Biomed Res. Int. 2019, 2019, doi:10.1155/2019/6462472.

19. Luken, M.; Sammons, A. Systematic Review of Mindfulness Practice for Reducing Job Burnout. Am. J. Occup. Ther. 2016, 70, p1-7002250020, doi:10.5014/ajot.2016.016956.

20. Connor, K.M.; Davidson, J.R.T. Development of a new Resilience scale: The Connor-Davidson Resilience scale (CD-RISC). Depress. Anxiety 2003, 18, 76-82, doi:10.1002/da.10113.

21. Laird, K.T.; Lavretsky, H.; St. Cyr, N.; Siddarth, P. Resilience predicts remission in antidepressant treatment of geriatric depression. Int. J. Geriatr. Psychiatry 2018, 33, 1596-1603, doi:10.1002/gps.4953.

22. Delgado-Gallegos, J.L.; Avilés-Rodriguez, G.; Padilla-Rivas, G.R.; Ángeles Cosio-León, M.D. los; Franco-Villareal, H.; Zuñiga-Violante, E.; Romo-Cardenas, G.S.; Islas, J.F. CLINICAL APPLICATIONS OF MACHINE LEARNING ON COVID-19: THE USE OF A DECISION TREE ALGORITHM FOR THE ASSESSMENT OF PERCEIVED STRESS IN MEXICAN HEALTHCARE PROFESSIONALS. medRxiv 2020, 2020.11.18.20233288, doi:10.1101/2020.11.18.20233288.

23. Mahase, E. Covid-19: Pfizer and BioNTech submit vaccine for US authorisation. BMJ 2020, 371, m4552.

24. Marie, E.V.A.; Bloomberg, U.; Getty, V.I.A. COVID VACCINE RESULTS. Nature 2020, 587, 337-338.

25. Taylor, S.; Landry, C.A.; Paluszek, M.M.; Fergus, T.A.; McKay, D.; Asmundson, G.J.G. Development and initial validation of the COVID Stress Scales. J. Anxiety Disord. 2020, 72, 102232, doi:10.1016/j.janxdis.2020.102232.

26. Marsden, P.V.; Wright, J.D. Handbook of Survey Research; 2nd ed.; Emerald Publishing Limited, 2010; ISBN 978-1848552241.

27. Padilla-Rivas, G.R.; Delgado-Gallegos, J.L.; Montemayor-Garza, R.D.J.; Franco-Villareal, H.; CoiserLeón, M.D.L.Á.; Avilés-Rodriguez, G.; Zuñiga-Violante, E.; Romo-Cardenas, G.S.; Islas, J.F. Dataset of the adapted COVID STRESS SCALES for Healthcare professionals of the Northeast region of Mexico . Data Br. 2021, 106733, doi:10.1016/j.dib.2021.106733.

28. Baryah, A.N.S.; Midha, V.; Mahajan, R.; Sood, A. Impact of Corona Virus Disease-19 (COVID-19) pandemic on gastrointestinal disorders. Indian J. Gastroenterol. 2020, 39, 214-219, doi:10.1007/s12664-020-01071-6.

29. Bello-Chavolla, O.; Bahena-López, J.; Antonio-Villa, N.; Vargas-Vázquez, A.; González-Díaz, A.; Márquez-Salinas, A.; Fermín-Martínez, C.; Naveja, J.; Aguilar-Salinas, C. Predicting mortality due to SARS-CoV-2: A mechanistic score relating obesity and diabetes to COVID-19 outcomes in Mexico Omar. J Clin Endocrinol Metab. 2020, 105, 1-13.

30. Horesh, D.; Brown, A.D. Covid-19 response: Traumatic stress in the age of Covid-19: A call to close critical gaps and adapt to new realities. Psychol. Trauma Theory, Res. Pract. Policy 2020, 12, 331-335, doi:10.1037/TRA0000592.

31. Chanana, N.; Sangeeta Employee engagement practices during COVID-19 lockdown. J. Public Aff. 2020.

32. Friganoviü, A.; Selip, P.; Iliü, B.; Sediü, B. Stress and burnout syndrome and their associations with coping and job satisfaction in critical care nurses: A literature review. Psychiatr. Danub. 2019, 31, 21-31.

33. Carstensen, B.; Klusmann, U. Assertiveness and adaptation: Prospective teachers' social competence development and its significance for occupational well-being. Br. J. Educ. Psychol. 2020, 1-27, doi:10.1111/bjep.12377.

34. Park, C.L.; Russell, B.S.; Fendrich, M.; Finkelstein-Fox, L.; Hutchison, M.; Becker, J. Americans' 
COVID-19 Stress, Coping, and Adherence to CDC Guidelines. J. Gen. Intern. Med. 2020, 35, 22962303, doi:10.1007/s11606-020-05898-9.

35. Pedrozo-Pupo, J.C.; Pedrozo-Cortés, M.J.; Campo-Arias, A.; Lee, S.A.; Chersich, M.F.; Gray, G.;

Fairlie, L.; Eichbaum, Q.; Mayhew, S.; Allwood, B.; et al. Coronavirus Anxiety Scale: A brief mental health screener for COVID-19 related anxiety. Cad. Saude Publica 2020, 16, 2020, doi:10.1007/s10461-020-02897-z.

36. Secretaría de Salud Datos Abiertos - Dirección General de Epidemiología / Secretaría de Salud / Gobierno / gob.mx; 2020;

37. CDC COVID-19 Forecasts: Cases Available online: https://www.cdc.gov/coronavirus/2019ncov/cases-updates/forecasts-cases.html (accessed on Jan 17, 2021).

38. García-Peñalvo, F.J.; Corell, A.; Abella-García, V.; Grande, M. La evaluación online en la educación superior en tiempos de la COVID-19. Educ. Knowl. Soc. 2020, 21, 26, doi:10.14201/eks.23086.

39. Besser, A.; Lotem, S.; Zeigler-Hill, V. Psychological Stress and Vocal Symptoms Among University Professors in Israel: Implications of the Shift to Online Synchronous Teaching During the COVID19 Pandemic. J. Voice 2020, doi:10.1016/j.jvoice.2020.05.028.

40. Pearman, A.; Hughes, M.L.; Smith, E.L.; Neupert, S.D. Age Differences in Risk and Resilience Factors in COVID-19-Related Stress Ann. J Gerontol B Psychol Sci Soc Sci 2020, 1-10, doi:10.1093/geronb/gbaa120.

41. Jribi, S.; Ben Ismail, H.; Doggui, D.; Debbabi, H. COVID-19 virus outbreak lockdown: What impacts on household food wastage? Environ. Dev. Sustain. 2020, 22, 3939-3955, doi:10.1007/s10668020-00740-y.

42. Camargo, C.P.; Tempski, P.Z.; Busnardo, F.F.; Martins, M. de A.; Gemperli, R. Online learning and COVID-19: a meta-synthesis analysis. Clinics (Sao Paulo). 2020, 75, e2286, doi:10.6061/clinics/2020/e2286. 